\title{
Simulation Driven Development of a Novel Ultrasonic Liquid Level Sensor
}

\author{
Andreas Hauck ${ }^{1}$, Martin Meiler ${ }^{1}$ \\ ${ }^{1}$ SIMetris GmbH, Am Weichselgarten 7, 91058 Erlangen, Germany \\ andreas.hauck@simetris.de
}

\begin{abstract}
In this work a novel, highly robust liquid-level sensor system based on the fluid-solid interaction of mechanical ultrasonic solid waves with liquid media is developed. Here, already in the design phase the Finite Element (FE) based simulation of the different coupled field phenomena is taken into account. The focus of the simulation is to characterize propagable transmission modes in the sender / receivers and their efficient excitation using piezoelectric transducers. For the accurate determination of the piezoelectric material parameters a new parameter identification process is conducted. Finally, the possible accuracy of the overall system is quantified in a fully coupled simulation of the complete system.
\end{abstract}

Key words: liquid level sensor, ultrasonic waves, coupled finite element simulation, piezoelectric materials, parameter identification

\section{Introduction / Sensor Principle}

The continuous and accurate measurement of the fluid level in foamy and sloshing media is a challenging task, which is common e.g. in chemical and food industry. Especially when considering the latter one, contamination of the liquid has to be prevented at any cost, which is one of the main drawbacks of floater-based measurement devices, being very common due to the low product price. While there are many robust and contactless methods (see [1]), most of them loose reliability when froth / foam is present on top of the liquid. For example, radarand ultrasonic-based sensors suffer from the diffracted waves on the surface. Capacitive and pressure-based sensors are also influenced by the foam and sloshing in a negative direction.

Due to these facts, a novel dipstick-type sensor is developed (see Fig. 1), which utilizes the principle of mechanical waves propagating in solid media in the ultrasonic range. These waves then couple to the fluid medium via compressional waves. For the excitation and receiving of the signal, suitable piezoelectric transducers are utilized. By measuring the time of flight of the mechanical / compressional wave, one can calculate the liquid level if the speed of sound in the waveguides and the liquid is known.

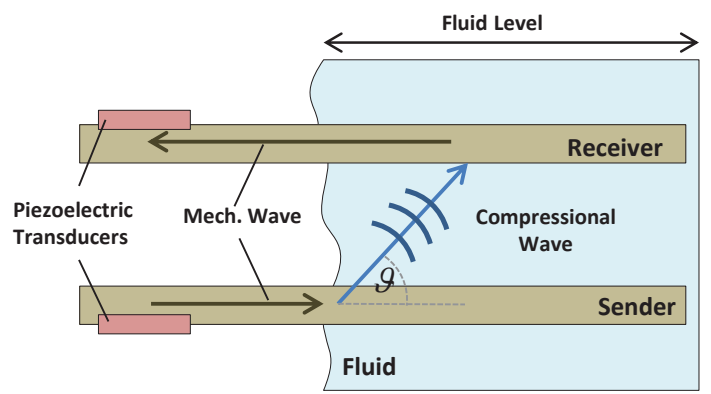

Fig. 1. Liquid level sensor with solid sender and receiver waveguides, driven by piezoelectric transducers.

The initial idea hereby is to involve the simulation already in the design phase to quickly vary and quantify many different designs. Specifically, the following aspects of the sensor development are considered in this work:

- Investigation of suitable solid wave types and their coupling to fluid

- Characterization of pre-selected piezoelectric transducers for generation / sensing of the waves

- Shape optimization of the mechanical waveguides

- Qualitative simulation of the complete sensor in a simplified 2D setup.

The idea of this paper is not to list all detailed results, but rather to focus on some of the main 
challenges within the design stages and development process. All simulation results were carried out using the coupled Finite Element software NACS [3].

\section{Propagable Waves and Coupling to Fluid}

As fluid media do not pose any resistance to shearing forces, only compressible waves can propagate in them with a fixed speed of sound $c_{\mathrm{f}}$, depending on the bulk modulus $K$ and the fluid density $\rho$

$$
c_{\mathrm{f}}=\sqrt{\frac{K}{\rho}} .
$$

In contrast, a solid medium has generally a shear-resistance, leading to more complex propagable waves. For unbounded media, there are two distinct wave types: One is a pure compressional wave with speed $c_{1}$, the other one a pure shear wave with a slower speed of sound $c_{\mathrm{t}}$.

For isotropic media, described by the elastic modulus $E$ and the Poisson ratio v, they can be computed as (see e.g. [2])

$$
\begin{aligned}
& c_{1}=\sqrt{\frac{(1-v) E}{(1+v)(1-2 v) \rho},} \\
& c_{\mathrm{t}}=\sqrt{\frac{E}{2(1+v) \rho}},
\end{aligned}
$$

which are related by the following inequality

$$
c_{1}>\sqrt{2} c_{\mathrm{t}} \text {. }
$$

In general media, both types of waves are present and can only be excited combined. Equations (2) and (3) are only valid for media with a dimension much larger than the corresponding wavelengths. In case that one or several dimensions of the body are comparable to the wavelength (e.g. plate, membrane), this dimension together with the boundary conditions (no stresses on the surface orthogonal to the waves) define the types of waves possible [7].

For thin plates these waves are called plate or Lamb waves, which can either be symmetric or antisymmetric. If the thickness $d$ is just a fraction of the longitudinal / transverse wavelength, basically only two modes are existing: the lowest antisymmetric $\left(\mathrm{A}_{0}\right.$-mode) and the lowest symmetric mode $\left(\mathrm{S}_{0}-\right.$ mode $)$, as depicted in Fig. 2. However, in contrast to unbounded media, Lamb waves exhibit a dispersive behavior, i.e. the speed of sound is not constant and in fact depends on the product

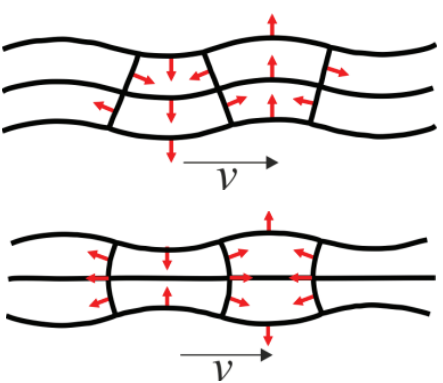

Fig. 2. Antisymmetric $A_{0}$ wave (top) and symmetric $S_{0}$ (bottom) plate wave.

of the thickness $d$ and frequency $\omega=2 \pi f$, as can be seen from the general dispersion relation, here just given for antisymmetric modes:

$$
\frac{\tan (\beta d / 2)}{\tan (\alpha d / 2)}=\frac{\left(k^{2}-\beta^{2}\right)^{2}}{4 \alpha \beta k^{2}}
$$

where

$$
\begin{aligned}
& \alpha=\omega^{2} / c_{l}{ }^{2}-k^{2} \\
& \beta=\omega^{2} / c_{t}{ }^{2}-k^{2},
\end{aligned}
$$

with $c_{1}$ and $c_{\mathrm{t}}$ from (2) and (3). It is obvious from (5) that the wave number $k$ depends on the frequency $\omega$ itself. In Fig. 3 the phase velocity is sketched for steel over the product of thickness and frequency, as obtained by numerical methods (see [8]). For increasing thickness, both lowest order modes approach a common constant phase velocity, which is exactly the socalled surface acoustic wave (SAW). This type of wave forms at the surface and decays exponentially in thickness [9].

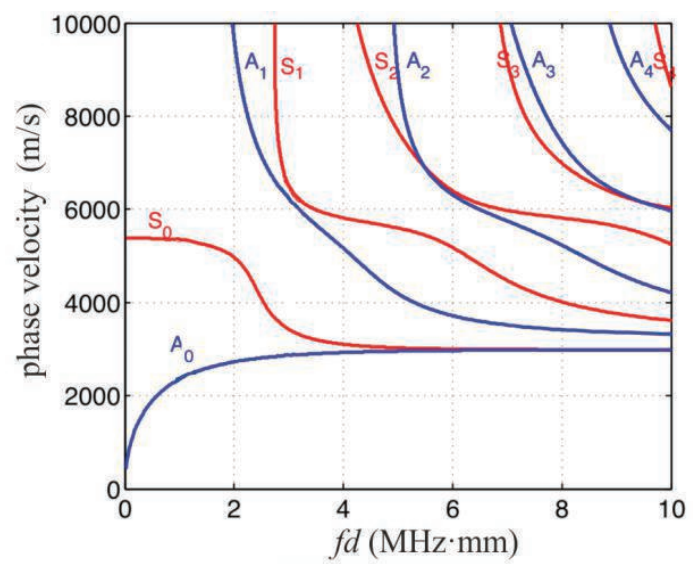

Fig. 3. Phase velocity of waves excited in a steelplate.

For the design of the waveguides for the sensor it is now mandatory to identify the waves excited by the transducers at hand (see following section). This can be performed by simulation of a single rod with the Finite Element (FE) method in frequency domain. To identify the resulting wave modes, we perform a 
spatial Fourier transform of the normal displacements on the surface of the rod

$$
\begin{gathered}
\mathcal{F} \\
u(x) \stackrel{(k)}{\Rightarrow} \\
k=1 / \lambda=f / c
\end{gathered}
$$

This delivers us the amplitude of the different waves propagating in the waveguide. For the initial investigation, we consider metallic waveguides, made of standard steel. The thickness $d$ is once chosen as $2 \mathrm{~mm}$ and for the other as $20 \mathrm{~mm}$. The excitation frequency here is $430 \mathrm{kHz}$, which is in the range of the resonance of the piezoelectric transducer investigated (see next section). The resulting mode spectrum is depicted in Fig. 4.
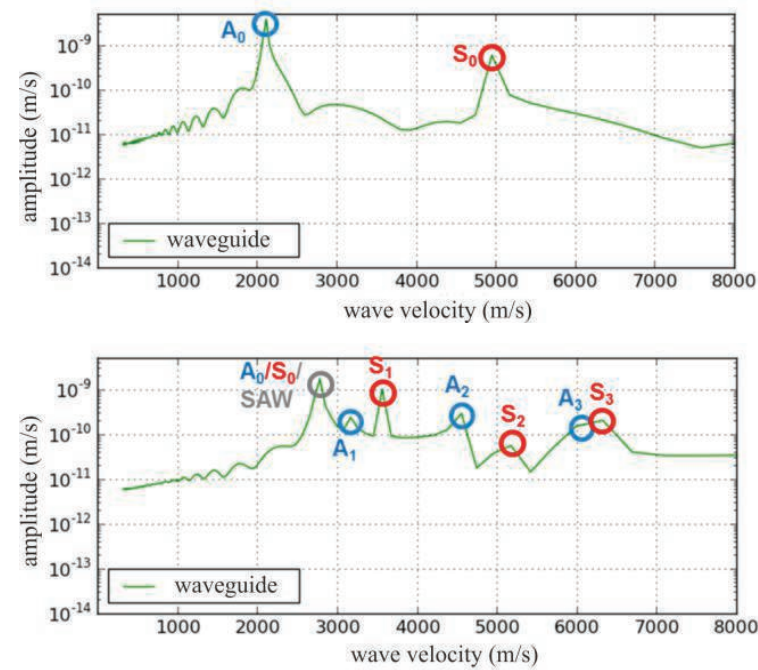

Fig. 4. Numerical wave spectrum for a $2 D$ rod with thickness $d=2 \mathrm{~mm}$ (top) and $d=20 \mathrm{~mm}$ (bottom). The corresponding dispersion curves are sketched in Fig. 3.

It can be recognized that within the thin rod of $\mathrm{d}=2 \mathrm{~mm}$ only the lowest order (anti-)symmetric modes are forming, which is also obvious from Fig. 3 as in this case the product $f d=0.86$ $\mathrm{MHz} \cdot \mathrm{mm}$. In contrast, for the thick rod d=20 mm the product $f d=8.6 \mathrm{MHz} \cdot \mathrm{mm}$ allows the propagation of modes up to 3rd order. Also due to the increased thickness - the $A_{0^{-}}$and $S_{0^{-}}$ modes cannot be distinguished, as in fact a surface acoustic wave (SAW) is forming, which exponentially decays in thickness direction.

In case the waveguide is subjected to fluid loading, the solid wave(s) couple their energy via mode conversion to a compressional wave into the fluid. This occurs at a characteristic angle $\vartheta$ (see Fig. 5)

$$
\cos (\vartheta)=c_{\text {water }} / \mathrm{c}_{\text {solid }}<1 \text {. }
$$

In case of the steel setup, all mechanical modes can couple to water. For other materials or thinner waveguides however, this condition may not be met, so there will be no travelling compressional wave forming in the fluid.

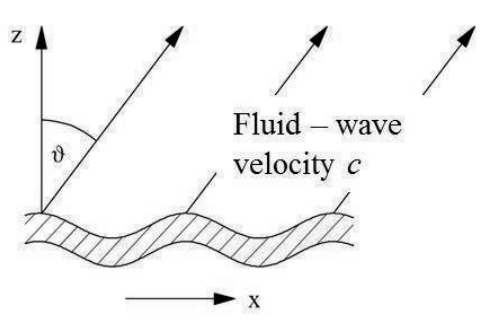

Fig. 5. Coupling of mechanical (plate) wave to fluid under characteristic angle [9].

\section{Transducer Characterization - Inverse Identified Material Parameters}

For the excitation of mechanical waves there exists a variety of different mechanisms [7]. Especially for the excitation of surface acoustic waves, we refer e.g. to [6].

In this project, there was already a dedicated piezoelectric transducer available (see Fig. 6), which is composed of PIC-255, a leadzirconate-titanate material. The transducer is polarized in thickness direction and is contacted with a wrap-around electrode of CuNi.
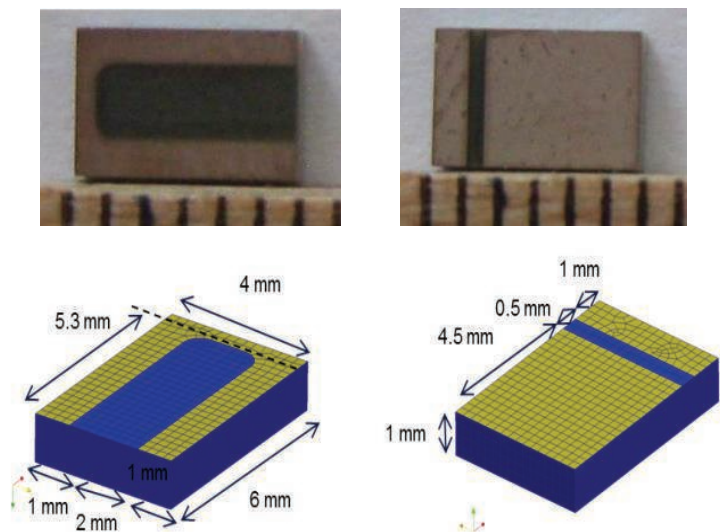

Fig. 6. Pictures (top) and FE model (bottom) for piezoelectric transducer with wrap-around electrode.

Since the transducer plays a key role in the overall system characterization, it is mandatory to have accurate mechanical material data available in order to guarantee reliable simulation results. As the manufacturer provided data is often measured using a simplified approach (e.g. IEEE / ANSI standard of measuring mono-modal vibrations) and in addition production parameters vary from badge to badge, we perform initially an inverse method to identify an improved set of material parameters. For details of this method, we refer to [4].

The idea of this method is to use the initial material data within a FE-simulation of a few different samples (see Fig. 7). The results of the 
simulation - the electric impedance as well as mechanical deformations - are compared to measurement results. If the deviation is above a given threshold, the material parameters are optimized in an inverse identification method, which results in an improved set of parameters (i.e. electrical permittivity $[\varepsilon]$, mechanical stiffness [c], piezoelectric coupling parameter $[\mathbf{e}]$ and scalar damping parameter $\alpha$ ). These parameters serve as input for the next iteration loop, until convergence is achieved. In [8] it is shown that single parameters can have deviations up to $20 \%$ compared to the manufacturer data.

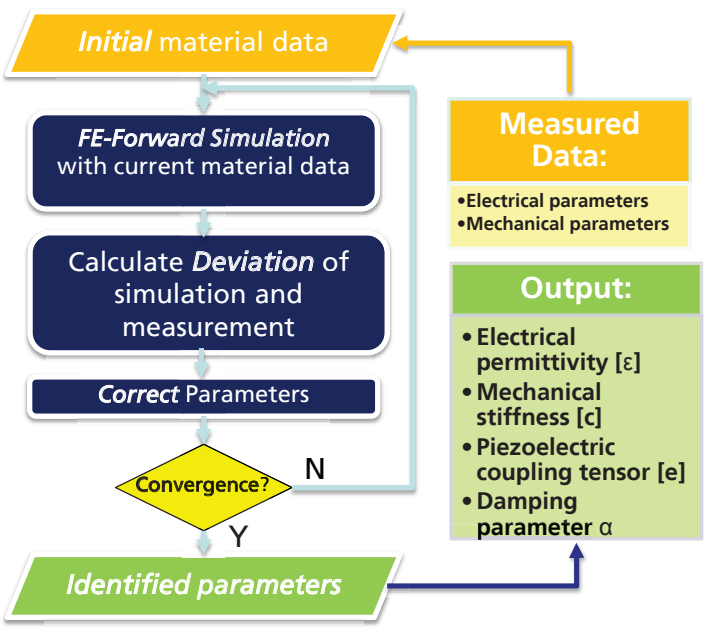

Fig. 7. Piezo-parameter identification process.

Using the improved set of material data, the impedance curve of the free transducer was determined and compared to measurements (see Fig. 8). It can be recognized that all the inplane modes up to $600 \mathrm{kHz}$ match very well, both in resonances and amplitude. Only the quasi-thickness modes around $1 \mathrm{MHz}$ show small deviations.

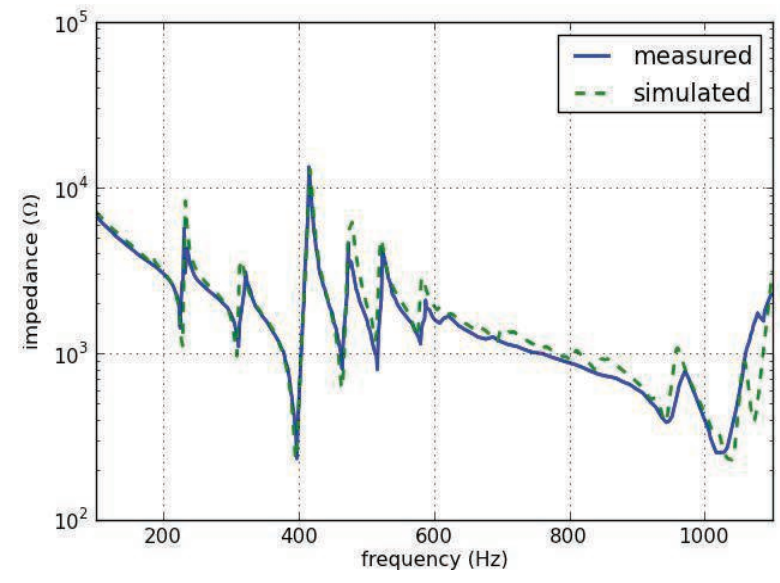

Fig. 8. Measured and simulated impedance spectrum of piezoelectric finger transducer.

In a second step, the effect of bonding the transducer onto a semi-infinite half-sphere is investigated using a FE model (see Fig. 9). The glue layer is assumed to be very thin (approx. $20 \mu \mathrm{m}$ ) and for the damping of the emitting solid waves, a so-called perfectly matched layer (PML) is utilized [2]. This allows us to truncate the propagation domain in the very near field.

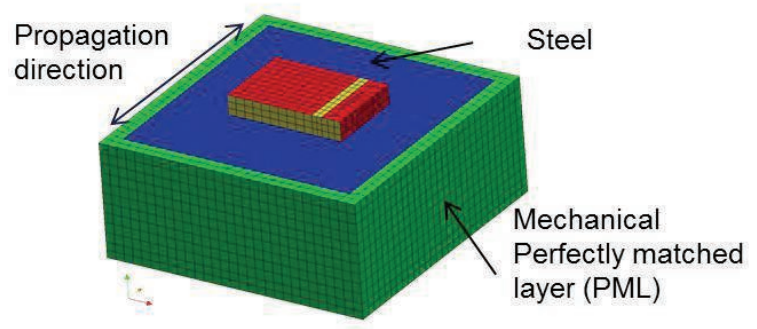

Fig. 9. FE model of bonded transducer on infinite volume with special damping layer (PML) [2].

From the results in Fig. 10 we can deduce, that the overall stiffness of the assembly is increased due to the surface bonding. In fact, the previous in-plane mode of $396 \mathrm{kHz}$ is now shifted to $426 \mathrm{kHz}$, resulting in a bending deformation. This is also the excitation mode which will be utilized in subsequent investigations.

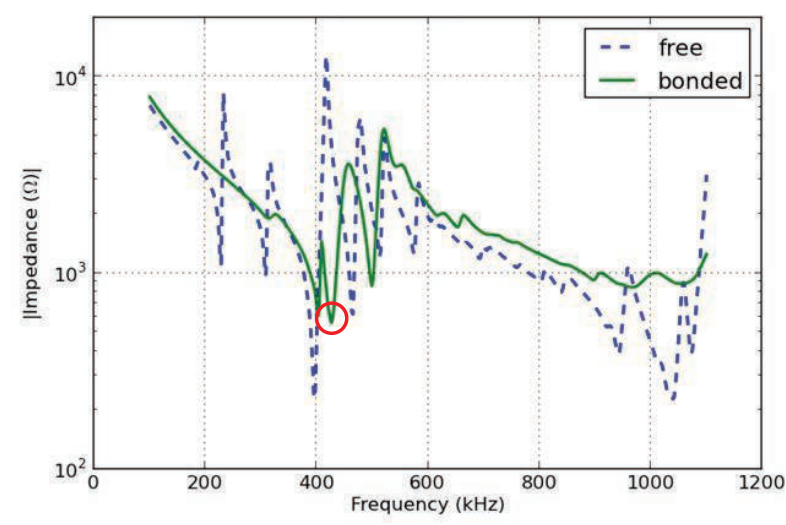

Fig. 10. Simulated impedance curves of free and bonded piezoelectric transducer.

In view of the previous section, one can utilize a different excitation method to "tune" the excitation to excite only a specific wave type using piezoelectric active waver sensors (PWAS) as proposed in [5]. The idea here is to use very thin piezo patches with full electrodes, bonded perfectly onto a structure. If the dimension of the piezo matches the wavelength of a specific (anti-)symmetric wave, the amplitude of this mode can be magnified, as the patch can be viewed as an idealized Dirac-like shear force at its edges. However, as the achievable amplitudes are much smaller than compared to the transducer used in this work, this approach was not investigated further. 


\section{Shape Optimization of Waveguides}

Another aspect in the design phase is to optimize the acoustic energy coupling from the sender waveguide to the receiving one, which can be quantified by means of the acoustic power $P_{\text {acou }}$

$$
P_{\text {acou }}=\int_{A} p \vec{v} \cdot \mathrm{d} \vec{A} .
$$

The related model for this investigation is sketched in Fig. 11. Due to the need for a 3dimensional solid-fluid model and the involved discretization effort, we truncate the domain using both, an acoustic perfectly matched layer for the fluid domain, as well as a mechanical one at the end of the waveguide to prevent artificial reflections. As excitation, a harmonic cW signal with $20 \mathrm{~V}_{\mathrm{pp}}$ was applied to the piezoelectric transducer at $426 \mathrm{kHz}$, which corresponds to the previously determined bending resonance mode.

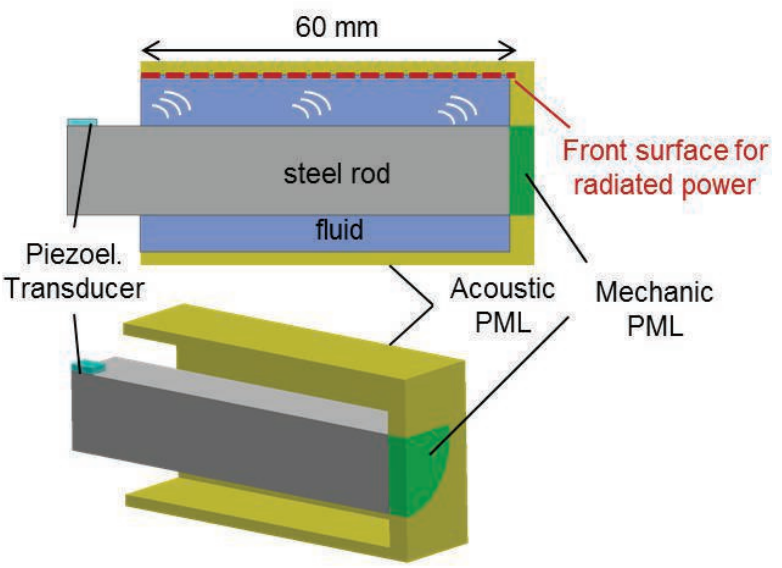

Fig. 11. Setup for calculation of radiated acoustic power.

We investigated solid, as well as hollow waveguides with different cross-sections, as depicted in Fig. 12. For the hollow structures, we expect the forming of the aforementioned plate waves.

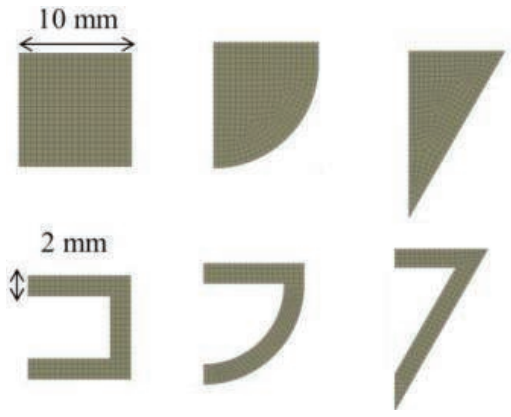

Fig. 12. Investigated cross-sections of mechanical waveguide.

The general observation in the investigation was that the solid waveguides (Fig. 12, top row) produce in general less acoustic power, which is due to the multitude of interfering waves generated in the rod. From the hollow structures, the semi-circular one has the best total radiated power and also radiates the largest part via the front surface (see Table 1).

Tab.1: Radiated acoustic power and relative amount on front surface for two selected crosssections.

\begin{tabular}{|c|c|c|}
\hline & $\begin{array}{c}\text { Radiated } \\
\text { acoustic power }\end{array}$ & $\begin{array}{c}\text { Ratio radiated } \\
\text { power front / total }\end{array}$ \\
\hline & $0.37 \mathrm{~mW}$ & $31.7 \%$ \\
\hline & $2.4 \mathrm{~mW}$ & $50.2 \%$ \\
\hline
\end{tabular}

From Fig. 13 one can also deduce that the solid, rectangular-shaped waveguide has an omnidirectional radiation pattern, whereas the hollow one radiates focused towards the receiving waveguide.

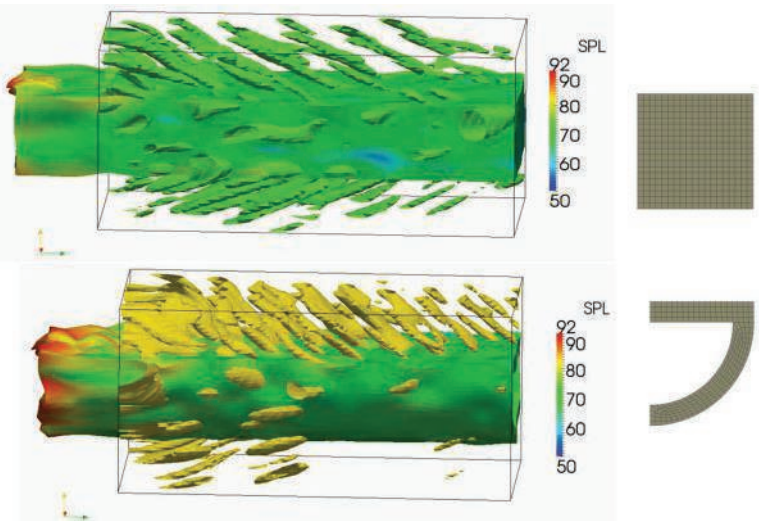

Fig.13. Comparison of pressure field for solid (top) and hollow semi-circular (bottom) shape of waveguide.

\section{Quantification of the Overall System}

Finally, the overall system behavior of the complete setup gets quantified, i.e. we consider a setup as sketched in Fig. 14, consisting of two waveguides of length $29.5 \mathrm{~cm}$ to measure a fluid level in the range of $2-20 \mathrm{~cm}$. The excitation of the sending transducer is a sinusoidal burst of $426 \mathrm{kHz}$ with 10 periods and a peak voltage of $20 \mathrm{~V}_{\mathrm{pp}}$. 


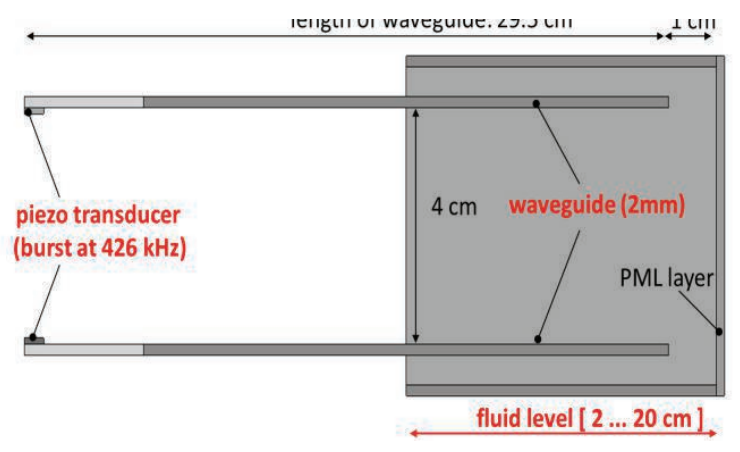

Fig. 14. Simulation setup for fluid-level measurement.

In Fig. 15 the electric voltage at the receiving transducer is drawn for a fluid level of $12 \mathrm{~cm}$, as well as the acoustic power transmitted from sender to receiver.
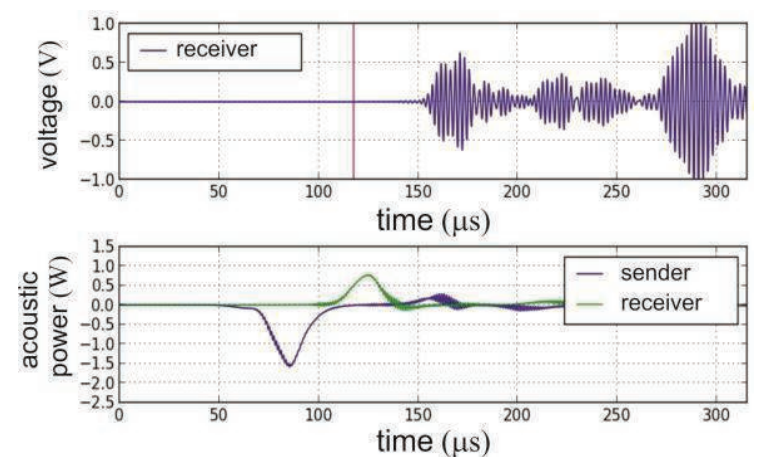

Fig. 15.Electric voltage at receiver (top) and transmitted acoustic power (bottom) for a fluid level of $12 \mathrm{~cm}$

In a last step, we perform a simple thresholdbased evaluation of the receiving voltage. In Fig. 16 the results are shown for different threshold levels $0.001-1 \mathrm{mV}$, in addition to the analytically calculated time of flight. For all the levels there is an almost linear relation between the fluid level and time of flight. Only for the largest threshold level of $1.0 \mathrm{mV}$ and small fill levels, there are some oscillations, so it might be better so reduce the threshold or resort to more robust algorithms.

\section{Conclusion}

In this work the simulation-based development steps of a liquid level sensor have been sketched. Especially the characterization of the generated plate waves, as well as the accurate simulation of the piezoelectric transducer were in the focus of this work. The FE simulation allows one also to simulate to the fully coupled piezo-mechanic-acoustic problem to quantify the final measurement effect before assembling a first prototype. Of course, further measurements on the prototypes have to investigate some effects in more detail, e.g. robustness in case of foam or sloshing media.

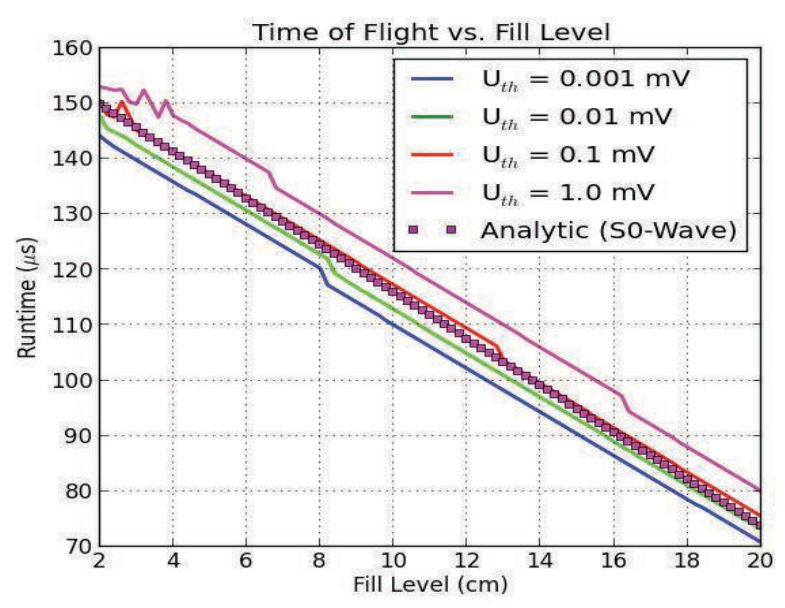

Fig. 16. Time of flight versus fluid level for different threshold levels of the receiving transducer.

\section{Acknowledgements}

This work is part of the project "FüSSE Füllstandssensor für schwierigste Messaufgaben" within the cluster Sensorik funded by the Bavarian Ministry of Economic Affairs and Media, Energy and Technology, which is greatly acknowledged.

\section{References}

[1] E. Amberger, Füllstandsmeßtechnik: Grundlagen und Anwendungsbeispiele, Verlage Moderne Industrie (1999)

[2] M. Kaltenbacher, Numerical Simulation of Mechatronic Sensors and Actuators, 2nd edition, Springer (2007)

[3] SIMetris GmbH, NACS 2.0 User Manual, 2014

[4] S. Rupitsch, R. Lerch, Inverse method to estimate material parameters for piezoceramic disc actuators, Applied Physics A: Materials Science \& Processing 97, 735-740 (2009); doi: 10.1007/s00339-009-5438-1

[5] V. Giurgiutiu, Tuned Lamb Wave Excitation and Detection with Piezoelectric Wafer Active Sensors for Structural Health Monitoring, Journal of Intelligent Material Systems and Structures 16, 291-305 (2005); doi: $10.1177 / 1045389 \times 05050106$

[6] G. Lindner, Sensors and actuators based on surface acoustic waves propagating along solidliquid interfaces, Journal of Physics D: Applied Physics 41, 1-13 (2008); doi: 10.1088/0022$3727 / 41 / 12 / 123002 \mid$

[7] J. L. Rose, Ultrasonic Waves in Solid Media, Cambridge University Press (1999)

[8] R.D. Mindlin, Waves and vibrations in isotropic elastic plates, in First Symposium on Structural Mechanics 1958, edited by J.N. Goodier and N. Hoff, Pergamon Oxford(1960)

[9] R. Lerch, S. Sessler, D. Wolf, Technische Akustik, 1st edition, Springer (2009) 